\title{
B cell profiling in malaria reveals expansion and remodeling of CD11c ${ }^{+}$ B cell subsets
}

\author{
Christopher Sundling, ${ }^{1,2}$ Caroline Rönnberg, ${ }^{1,3,4}$ Victor Yman, ${ }^{1}$ Muhammad Asghar, ${ }^{1,2}$ \\ Peter Jahnmatz, ${ }^{1,5}$ Tadepally Lakshmikanth, ${ }^{6}$ Yang Chen, ${ }^{6}$ Jaromir Mikes, ${ }^{6}$ Mattias N. Forsell, ${ }^{7}$ \\ Klara Sondén,, Adnane Achour,, 1,2,8 Petter Brodin, ${ }^{6,9}$ Kristina E.M. Persson, ${ }^{3,10}$ and Anna Färnert ${ }^{1,2}$ \\ 'Division of Infectious Diseases, Department of Medicine Solna, Karolinska Institutet, Stockholm, Sweden. 'Department \\ of Infectious Diseases, Karolinska University Hospital, Stockholm, Sweden. ${ }^{3}$ Department of Microbiology, Tumor, and Cell \\ Biology, Karolinska Institutet, Stockholm, Sweden. ${ }^{4}$ Department of Clinical Microbiology, Karolinska University Hospital, \\ Huddinge, Stockholm, Sweden. ${ }^{5}$ Mabtech AB, Stockholm, Sweden. '5Science for Life Laboratory, Department of Women's \\ and Children's Health, Karolinska Institutet, Solna, Sweden. 'Division of Infection \& Immunology, Department of Clinical \\ Microbiology, Umeå University, Umeå, Sweden. ${ }^{8}$ Science for Life Laboratory, Department of Medicine Solna, Karolinska \\ Institutet, Stockholm, Sweden. ${ }^{9}$ Department of Newborn Medicine, Karolinska University Hospital, Solna, Sweden. \\ ${ }^{10}$ Department of Laboratory Medicine, Lund University, Skåne University Hospital, Lund, Sweden.
}

Humoral immunity is important in limiting clinical disease in malaria, yet the longitudinal B cell response to infection remains unclear. We performed a 1-year prospective study in patients treated for acute Plasmodium falciparum malaria for the first time or with previous exposure to the disease. Using an unbiased exploratory approach with mass cytometry, followed by targeted flow cytometry, we found that approximately $80 \%$ of mature $B$ cells that proliferated in response to acute infection expressed CD11c. Only approximately $40 \%$ of $C D 11 c^{+}$B cells displayed an atypical B cell phenotype, with the remaining cells primarily made up of activated and resting memory B cells. The CD11c B cells expanded rapidly following infection, with previous exposure to malaria resulting in a significantly larger increase compared with individuals with primary infection. This was attributed to an expansion of switched $\mathrm{CD} 11 \mathrm{c}^{+} \mathrm{B}$ cells that was absent in primary infected individuals. The rate

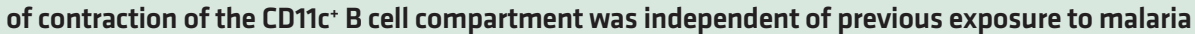
and displayed a slow decay, with a half-life of approximately $\mathbf{3 0 0}$ days. Collectively, these results identify CD11c as a marker of B cells responding to malaria and further highlight differences in primary and secondary $B$ cell responses during infection.

Authorship note: CS and CR contributed equally to this work.

Conflict of interest: The authors have declared that no conflict of interest exists.

Copyright: (c) 2019 American Society for Clinical Investigation

Submitted: November 27, 2018 Accepted: March 27, 2019 Published: May 2, 2019.

Reference information: /CI Insight. 2019;4(9):e126492. https://doi. org/10.1172/jici.nsight.126492

\section{Introduction}

Plasmodium falciparum malaria remains a major cause of morbidity and mortality, especially among children in sub-Saharan Africa (1). Repeated infections result in a slow acquisition of clinical immunity, with subsequent episodes typically presenting with milder symptoms and eventually protection against clinical disease (2). Parasite-specific antibodies play a key role in protection, as demonstrated in previous experiments with passive transfer of immunoglobulin from partially immune adults to children with malaria, who then managed to control their infection (3). To achieve clinical immunity it appears that a progressively broad and potent antibody response against parasite antigens is required $(4,5)$. However, even when such an antibody response is achieved, immunity wanes within a few years in the absence of reexposure (6), despite the presence of parasite-specific memory B cells (7).

Memory B cells specific to malaria parasite antigens are elicited at levels comparable to conventional licensed vaccines, such as the diphtheria toxoid vaccine (8), and can persist for prolonged periods of time in individuals both from endemic areas and in travelers contracting the infection for the first time (7). The B cell memory compartment can contain potent parasite-inhibiting specificities $(4,9)$. However, the presence of malaria-specific memory B cells by itself has not been found to protect the individual from $P$. falciparum infection or clinical disease (8). Impaired B cell development and function, associated with an expanded population of atypical B cells, have been proposed to explain the slow and incomplete immunity observed 
in malaria $(10,11)$. However, few studies have evaluated the effect of a single episode of malaria on the long-term dynamics of atypical B cell responses in humans, especially in the context of de novo infection versus infection of individuals with previous exposure to the disease.

Although the mechanisms behind atypical B cell generation remain unclear, recent studies in humans (12) and mice (13) suggest that the strong skewing of the malaria-specific immune response toward a $\mathrm{T}$ helper type 1 (Th1) profile could have negative effects on the reactivation, development, and expansion of long-lived $\mathrm{B}$ cell responses in malaria. The extensive induction of Th1 responses following infection is associated with an increase in proliferation of primarily an atypical subpopulation of memory B cells devoid of the classical surface markers CD21 and CD27 $(14,15)$. These atypical cells display an RNA expression profile $(11,15)$ and surface marker profile (16-18), which diverges from that of both resting memory and naive B cells. They have also typically been shown to express high levels of the surface markers FcRL5 and CD11c $(11,16,18)$.

The unique phenotype of atypical B cells suggests that their function could differ from that of conventional B cell responses. In addition, the atypical B cells are enriched for self-reactive B cell clones (19); however, it remains unclear if they contribute to the circulating antibody pool, as they display reduced responsiveness to restimulation with $\mathrm{B}$ cell receptor $(\mathrm{BCR})$ and $\mathrm{T}$ helper signals in vitro $(11,18)$. In mice, a phenotypically similar B cell subset, expressing CD11c, was shown to have potent antigen-presenting functions (20). They were also involved in autoantibody-mediated destruction of red blood cells in a mouse model of malaria (21), indicating that these cells could have important functions during infection.

Atypical B cell numbers are elevated in individuals living in malaria-endemic areas $(16,22-24)$ and expand with recurrent parasite exposure (5). Accumulation of cells with a similar CD21- $\mathrm{CD} 27^{-}$phenotype and transcriptional profile have also been observed in individuals with chronic viral infections, such as HIV, bacterial infections, such as tuberculosis, as well as autoimmune disorders and primary immunodeficiencies $(18,25-32)$. Altogether, these studies suggest that atypical B cells are formed or accumulate in circulation as a response to prolonged infection and/or inflammation. However, the elicitation and dynamic regulation of these cells following acute infection remains to be fully understood.

In this study, our aim was to explore the B cell profiles and dynamics after a single episode of $P$. falciparum malaria. We evaluated the immune responses in malaria-infected travelers that returned to an area free of malaria, which enabled us to investigate the immune response longitudinally in the absence of parasite reexposure. Moreover, we compared the B cell dynamics in individuals with a primary infection with those with previous exposure to parasites. Using an unbiased exploratory approach with mass cytometry, followed by targeted flow cytometry, we found that CD11c was a selective marker for B cells that responded to the infection. Contrary to our expectations, CD11c was not only expressed by CD21-CD27- atypical B cells, but also by several other B cell subsets. Our analysis revealed that these cells were enriched for $P$. falciparum-specific B cell clones and that they contained both switched and unswitched B cells. The expansion of switched, but not unswitched, B cells was determined by previous parasite exposure, indicating a clear role for memory in driving the expansion of switched CD11 ${ }^{+} \mathrm{B}$ cells. Collectively, this study demonstrates the contribution of de novo and memory $\mathrm{B}$ cell responses to malaria and highlights the dynamic expression of several important markers in different B cell subsets.

\section{Results}

Previous exposure to malaria leads to enhanced parasite-specific antibody production upon infection. Here, we studied 51 individuals infected with $P$. falciparum malaria, who were followed prospectively with repeated sampling over 1 year (Figure 1). Seventeen individuals experienced their first episode of malaria, whereas 34 individuals had a previous history of repeated malaria exposure and, thus, experienced a recall response, with subsequent activation of malaria-specific memory responses. Peripheral blood mononuclear cells (PBMCs) were not isolated from 2 individuals, which explains the different number of included individuals in serological and flow cytometric assays (Figure 1). We defined the groups as "primary infected" and "previously exposed," respectively. In addition, 14 healthy individuals who reported that they had never traveled to malaria-endemic regions were sampled at 1 time point and included as a control group to indicate baseline levels of investigated cell populations. Importantly, none of the study participants had travelled to a country with endemic malaria during the 1-year follow-up. This study set-up allowed us to perform what we believe to be a unique and well-controlled study to dissect the dynamics of both primary and secondary immune responses to malaria without the risk of reexposure as a confounding factor. Overall, the general clinical characteristics of the acute response to the malaria episode were similar between the groups 

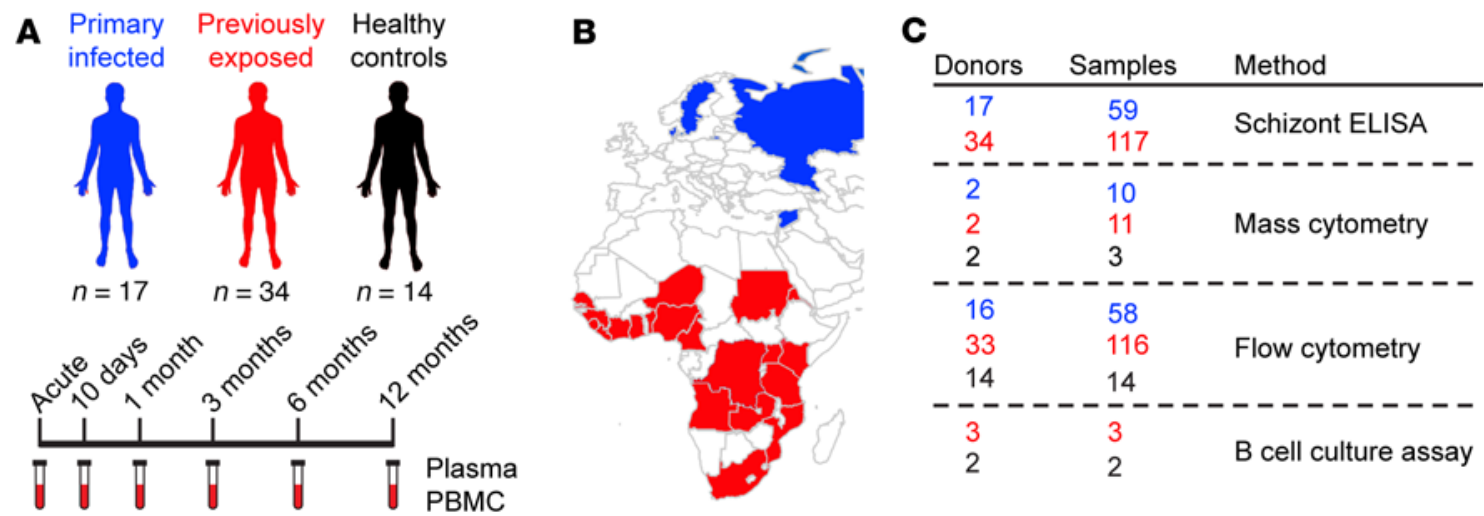

Figure 1. Schematic of study cohort and experimental setup. (A) The study cohort consisted of 3 groups, with individuals that had lived in malaria-endemic areas from child-hood (previously exposed, $n=34$ ), that were infected for the first time (primary infected, $n=17$ ), and healthy individuals with no history of travel to malaria-endemic areas (healthy controls, $n=14$ ). Individuals with malaria were sampled for peripheral blood at the acute infection and at 10 days and 1, 3, 6, and 12 months after acute infection. (B) Country of origin of previously exposed (red) and primary infected (blue) individuals. All healthy controls had Swedish origin. (C) The number of donors and samples from previously exposed (red), primary infected (blue), and healthy controls (black) used with each method.

(Supplemental Table 1; supplemental material available online with this article; https://doi.org/10.1172/ jci.insight.126492DS1). Following infection, both groups experienced a temporary reduction in circulating lymphocytes, while the levels of neutrophils and monocytes increased. As expected, white blood cell counts returned to normal values within 30 days after diagnosis and treatment (Supplemental Figure 1).

As an indication of previous malaria exposure, we asked how many years were spent in a malaria-endemic area and how long ago since the individual moved away from the endemic area. The median time living in an endemic area was 26 years (range 15-39 years, $n=34$ ), with a median time of 12 years (range 0-46 years, $n=34$ ) since the individual left (Supplemental Table 1). To determine if previous exposure to malaria generated B cell memory, we assessed schizont-specific IgG following infection (Figure 2). Consistent with a B cell memory response, we observed a more extensive schizont-specific IgG response in previously exposed individuals compared with those infected for the first time (5.5-fold difference, $95 \% \mathrm{CI}$ : $2.2-13.5, P=0.0005)$. These data corroborated our grouping of individuals based on previous exposure to malaria. The antibody responses in these individuals were further evaluated for a panel of merozoite antigens previously, indicating a broader and more long-lived response in previously exposed individuals compared with those with primary infection (33). The magnitude of the schizont-specific antibody response was not correlated with the time spent in or since leaving a malaria-endemic area $\left(\mathrm{r}^{2}=-0.04, P=0.95\right.$ and $\mathrm{r}^{2}=0.10, P=0.051$, respectively). There was, however, an effect of the time since symptom onset on early schizont-specific IgG antibody levels, but only in primary infected individuals $\left(\mathrm{r}^{2}=0.56, P=0.0032\right)$, explaining the high titers at the acute time point in some primary infected individuals (Figure 2A).

Phenotyping of the B cell compartment by mass cytometry. We used mass cytometry to characterize the phenotype of B cells in circulation during primary or secondary immune responses following $P$. falciparum infection. The main aim was to find markers of interest that could be used in a flow cytometry panel to identify key B cell populations affected by malaria. We assessed the expression pattern of 23 cell markers (Supplemental Table 2) in PBMCs longitudinally sampled over 1 year in 2 previously exposed and 2 primary infected individuals and 3 healthy controls sampled at 1 time point. The included individuals were randomly chosen, with the rationale that important cell populations should be present in all infected individuals. To enable an unbiased analysis of mass cytometry data, we selected $\mathrm{CD} 45^{+} \mathrm{CD} 3^{-} \mathrm{CD} 19^{+} \mathrm{B}$ cells and then used t-stochastic neighbor embedding (tSNE) to reduce dimensionality, followed by a graph-based clustering analysis (Figure 3A) (34). The data analysis resulted in the identification of $10 \mathrm{~B}$ cell clusters (Figure 3B), following exclusion of cells expressing the non-B cell markers CD11b, CD16, CD14, and CD3 or representing $<0.5 \%$ of analyzed cells (Supplemental Figure 2). The B cell clusters were further assessed for their contribution to the CD19+ $\mathrm{B}$ cell pool (Figure 3C) and analyzed for marker expression to determine the cell phenotype (Figure 3D).

Naive B cells, defined as $\mathrm{CD} 21^{+} \mathrm{CD} 27^{-} \mathrm{IgD}^{+}$, were found in cluster 10 , which was the largest cell cluster, corresponding to $31 \%$ of B cells. Memory B cells, defined as CD $21^{+} \mathrm{CD} 24^{+} \mathrm{CD} 27^{+}$, constituted 3 clusters (clusters 3, 8, and 11). These clusters were further defined by BCR isotype (Figure 3D). 
A

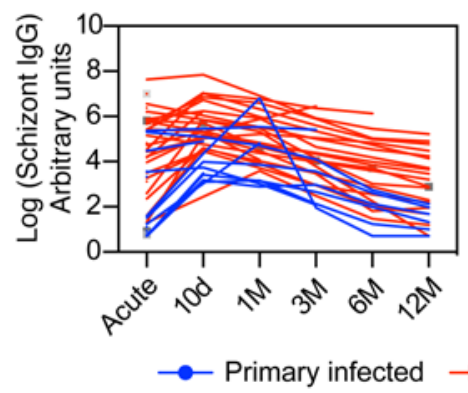

B

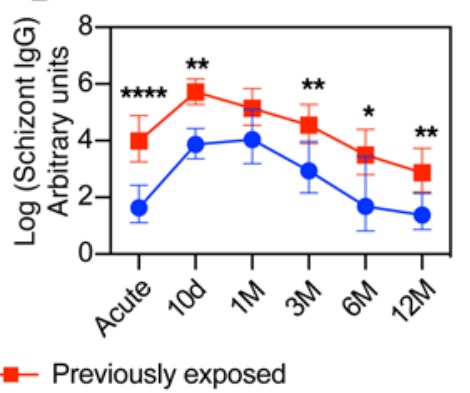

Figure 2. P. falciparum schizont IgG antibody dynamics. (A) Relative schizont IgG titers for each individual donor. (B) Geometric mean relative antibody titers $\pm 95 \% \mathrm{Cl}$ grouped as individuals with a primary infection (blue circles, $n=17$ ) or with previous exposure (red boxes, $n=33$ ). All antibody titers are log transformed. Differences between the groups were evaluated by a linear mixed-effects model with restricted maximum likelihood followed by an LS means Student's $t$ test for specific comparisons. ${ }^{*} P<0.05,{ }^{* *} P<0.01,{ }^{* * *} P<0.0001$.

Cluster 11 displayed lower expression of CD27 compared with clusters 3 and 8. Closer examination at the single-cell level revealed that the majority of cells were negative for CD27 expression (Figure 3E). Considering the lack of expression of $\operatorname{IgD}$, IgM, and IgA, these cells likely represent a switched CD27 memory B cell subset. Such memory B cells have been identified previously (35) and show similar BCR characteristics to $\mathrm{CD} 27^{+}$memory B cells (36). Cluster 19, which contributed $18.7 \%$ of the cells expressed IgM and IgD, but lacked expression of CD21 and CD27. This cluster may represent transitional B cells but also demonstrated low expression of CD24 and CD38, which contrasts with previously published data on transitional B cells (37). Hence, further analysis is required to fully understand the composition of this cluster. Clusters 16, 17, and 21 were characterized by high expression of CD11c. Clusters 16 and 17 were further defined by lack of $\mathrm{CD} 21$ and CD27, likely representing atypical B cells, while cluster 21 expressed detectable levels of CD27. Further investigation of CD27 expression within this cluster at the single-cell level showed that cluster 21 was composed of a mix of $\mathrm{CD} 27^{+}$activated memory B cells and CD27- atypical B cells (Figure 3E). Interestingly, among CD11 ${ }^{+} \mathrm{B}$ cells, it was primarily cluster 21 that had increased in previously exposed individuals compared with primary infected individuals and healthy controls (Figure 3C). Cluster 17 was further defined by high expression of $\mathrm{IgD}$, while cluster 16 was negative for IgD, IgM, and IgA and, most likely, represented IgG-switched cells. Plasmablasts, defined as B cells with low expression of CD20 and high expression of CD38, were composed of 2 clusters (clusters 14 and 15) that could be separated by variable expression of CD27 (Figure 3D).

We analyzed the contribution of these cell populations to the total B cell compartment at each sample time point during and after the acute malaria episode and compared with samples from healthy controls $(n=$ 3) (Figure 3F). Naive and memory B cell frequencies were similar to healthy controls at the acute time point, while plasmablasts and $\mathrm{CD} 11 \mathrm{c}^{+} \mathrm{B}$ cells had expanded in most donors. Naive B cells seemed to increase in individuals with primary infection, while memory frequencies increased over time in both groups. As expected, the plasmablast expansion was transient and had returned to baseline frequency already 10 days after the acute infection. In contrast $\mathrm{CD} 11 \mathrm{c}^{+} \mathrm{B}$ cells remained in circulation for more than 3 months in previously exposed individuals, potentially indicating a differential regulation of this compartment in response to malaria.

Effect of previous malaria exposure on $B$ cell dynamics. Following the identification of markers associated with differential B cell regulation between the groups by mass cytometry, we expanded the analysis to include samples from 63 individuals (primary infection: $n=16$; previously exposed: $n=33$; healthy controls: $n=14$ ) (total $n=189$ samples) using selected markers in flow cytometry. Longitudinal B cell dynamics following malaria were evaluated from isolated PBMCs using a 13-color flow cytometry panel optimized for evaluation of B cell subsets and including CD11c in addition to other markers associated with B cell differentiation (Supplemental Table 3). Cells were first gated for lymphocytes by forward and side scatter followed by singlets and live cells (Supplemental Figure 3). B cells were thereafter gated either as $\mathrm{CD} 19^{+} \mathrm{CD} 20^{+}$cells followed by $\mathrm{CD} 10$ to distinguish mature $\left(\mathrm{CD} 10^{-}\right)$and immature $\left(\mathrm{CD} 10^{+}\right) \mathrm{B}$ cells or $\mathrm{CD} 19^{+} \mathrm{CD} 20^{\text {lo }}$ cells (Figure $4 \mathrm{~A}$ ). $\mathrm{CD} 19^{+} \mathrm{CD} 20^{\text {lo }}$ cells were further gated for $\mathrm{CD} 38^{\text {hi }} \mathrm{CD} 27^{+}$cells, corresponding to plasmablasts. As there was no difference in lymphocyte counts between the groups during the 
A

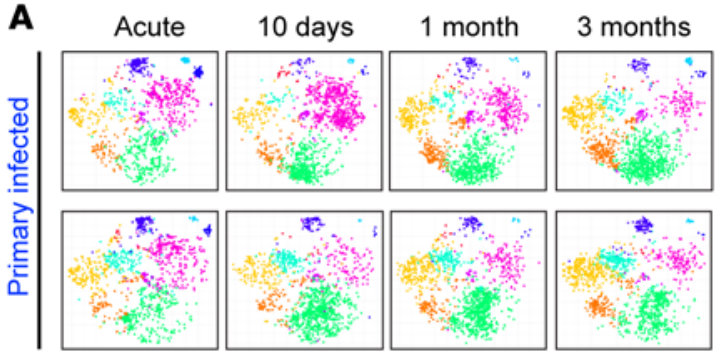

6 months 12 months
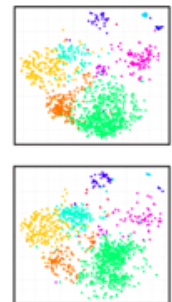

B

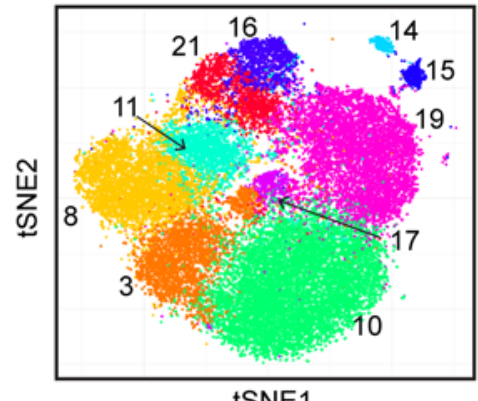

tSNE1
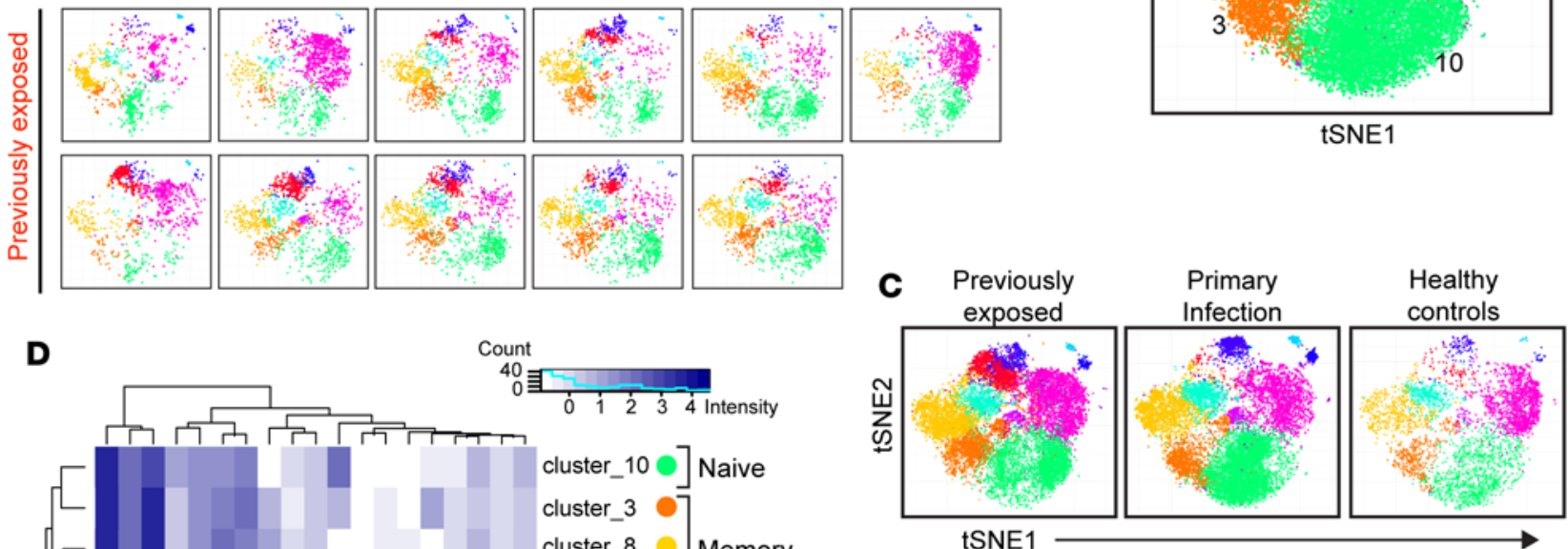

D

ount

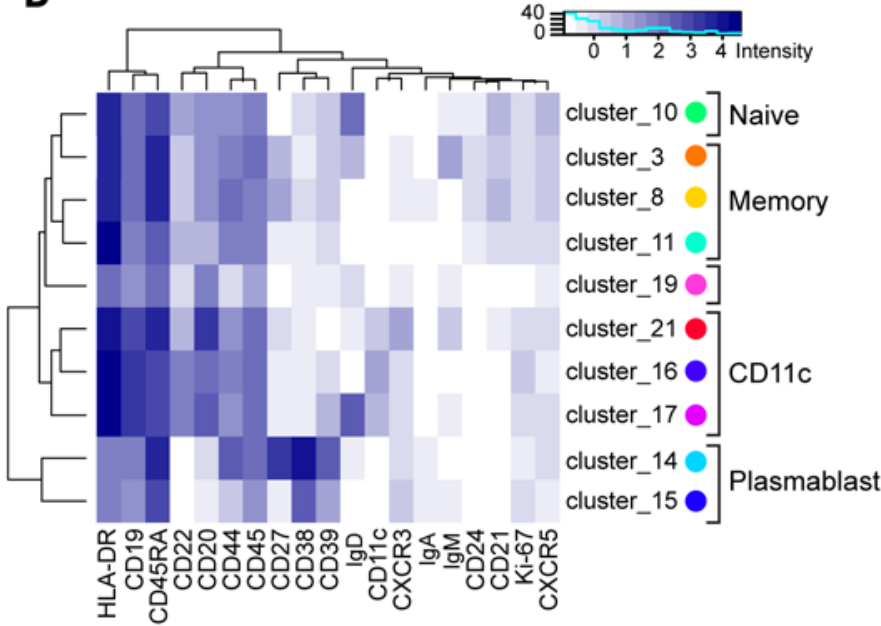

E
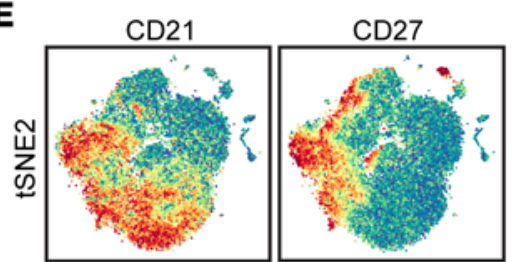

CD11c

tSNE1

$\mathbf{F}$

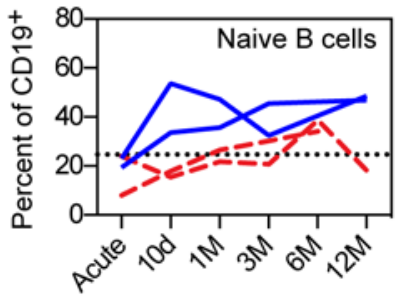

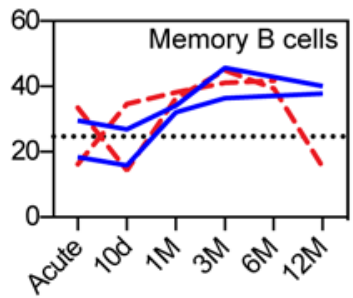

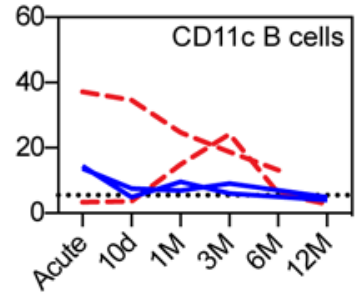

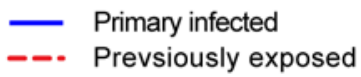

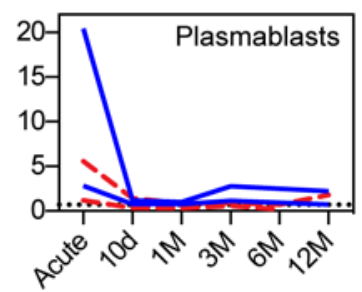

Figure 3. B cell phenotypic analysis by mass cytometry. (A) tSNE analysis followed by clustering of mass cytometry data for individual primary infected ( $n$ $=2$ ) and previously exposed $(n=2)$ donors. (B) Merged tSNE plot for all donors and time points $(n=24$ samples), with each cluster indicated by number and color. (C) Merged tSNE plots for each group (previously exposed: $n=11$ samples; primary infection: $n=10$ samples; healthy controls: $n=3$ samples). (D) Heatmap showing marker expression within each cluster. Circles are colored as cluster plots. (E) Expression of CD21, CD27, and CD11c for analyzed cells. (F) Contribution of each B cell subset to total $C D 19+B$ cells at different time points after infection. Blue solid lines indicate individuals with a primary infection; red dashed lines indicate individuals previously exposed to malaria. The dotted black line indicates the mean frequency of each population in healthy controls.

study follow-up (Supplemental Figure 1; 1.0-fold difference, 95\% CI: 0.7-1.5, $P=0.91$ ), changes in cell frequencies could be compared directly. However, for completion, all data were also normalized to total lymphocyte numbers (Supplemental Figure 4). As expected, both approaches produced similar results.

Plasmablast frequencies, normalized to total B cell numbers, were expanded in both malaria-infected groups compared with healthy controls at the time of acute infection (primary infected: 11.1-fold increase, 95\% CI: 4.2-30.1, $P<0.0001$ and previously exposed: 8.3 -fold increase, 95\% CI: 3.5-19.9, 
A
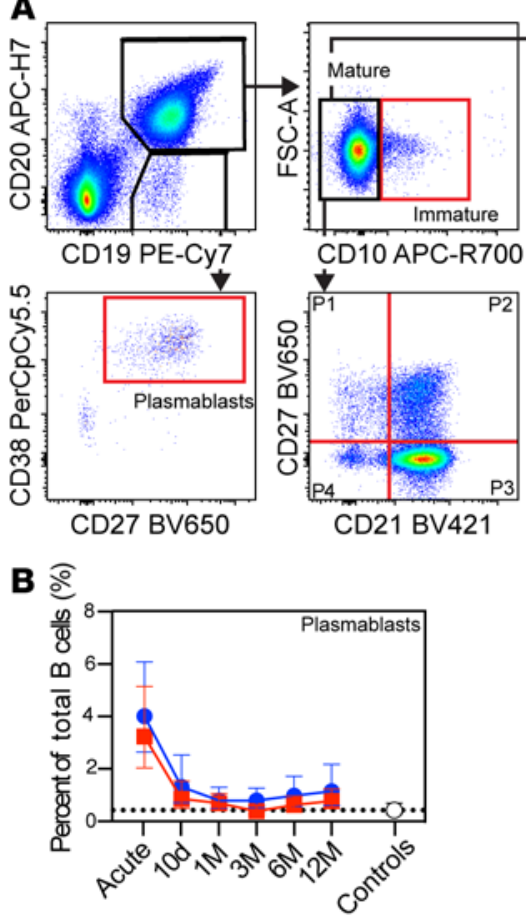

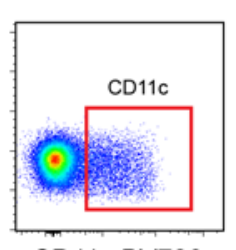

CD11c BV786
C
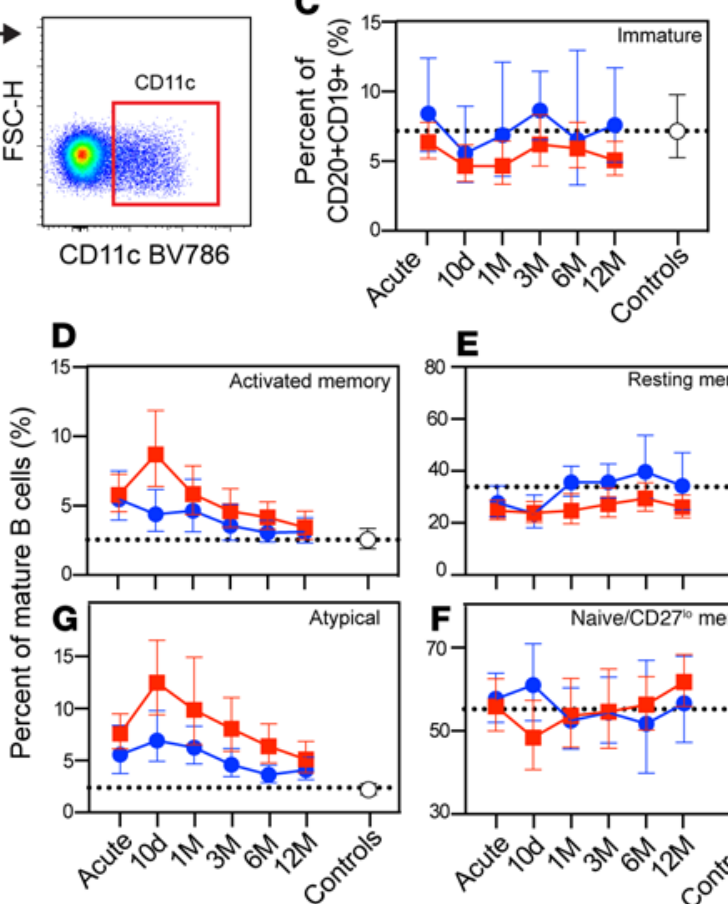

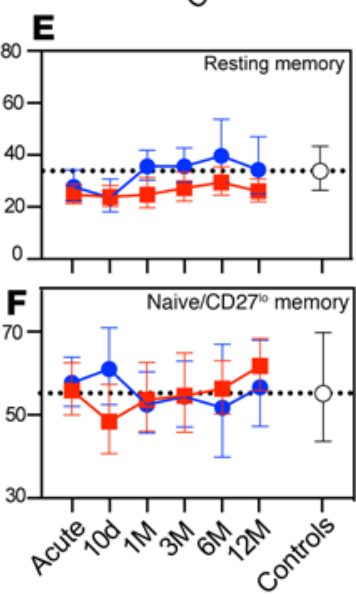

H

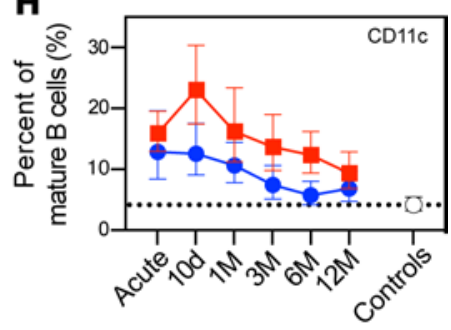

I

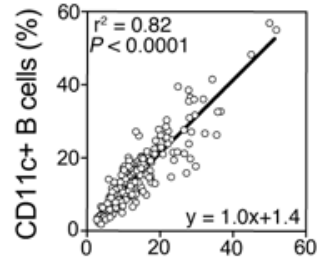

CD21lo B cells (\%)

J

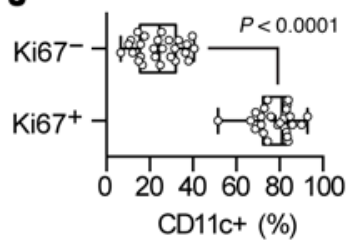

Primary infected

- Previously exposed $-\circ-$ Controls

Figure 4. B cell population dynamics by flow cytometry. (A) Gating strategy of B cell subsets. Cells were pregated for size, singlets, and live cells. B cells were identified by $C D 19$ and $C D 20$ expression. $C D 19+C D 20^{10} B$ cells were further gated for $C D 27$ and $C D 38$ for definition of (B) plasmablasts. $C D 19+C D 20^{+} B$

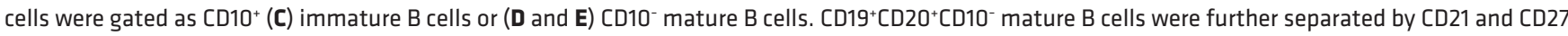
for activated memory (D), resting memory (E), naive/CD27/0 memory (F), and atypical (G) B cells or (H) CD11c B cells. Primary infected individuals (blue circles, $n=16$ ) were compared with previously exposed (red boxes, $n=32$ ) and noninfected healthy controls (white circles, $n=14$ ). Data are shown as geometric mean $\pm 95 \% \mathrm{Cl}$. Dotted lines correspond to the healthy control geometric mean from a single time point. (I) Mature CD11c $\mathrm{B}$ cells were correlated with CD21 $1^{10} \mathrm{~B}$ cells $(n=175)$, corresponding to activated memory and atypical B cells, using linear regression with calculation of Pearson $\mathrm{r}^{2}$. (J) Ki67 ${ }^{+}$and Ki67 ${ }^{-}$mature B cells were compared for frequency of CD11 $\mathrm{C}^{+} \mathrm{B}$ cells $(n=26$; Wilcoxon matched pairs test).

$P<0.0001$ ) (Figure 4B). These populations then rapidly contracted within 10 days after treatment initiation, consistent with short-lived plasmablast dynamics. The rate of expansion and contraction of plasmablasts was similar in both infected groups.

The frequency of immature B cells, gated as $\mathrm{CD} 19^{+} \mathrm{CD} 20^{+} \mathrm{CD} 10^{+}$cells, was similar to that of healthy controls at any time point for either of the malaria-infected groups (Figure 4C). This indicated that the immature B cell compartment was not strongly affected by the current infection or by previous parasite exposure. Mature B cells $\left(\mathrm{CD} 19^{+} \mathrm{CD} 20^{+} \mathrm{CD} 10^{-}\right)$were separated into 4 populations $(\mathrm{P})$ based on expression of CD21 and CD27 (Figure 4A, bottom right, and Figure 4, D-G).

Activated memory B cells $\left(\mathrm{CD} 21^{-} \mathrm{CD} 27^{+}\right)$were elevated for both individuals with primary infection and previously exposed individuals compared with healthy controls at the acute time point (2.2-fold increase, 95\% CI: $1.2-3.7, P=0.0042$ and 2.3-fold increase, 95\% CI: $1.4-3.7, P=0.0004$, respectively) (Figure 4D). The activated memory B cell frequencies remained elevated until 3 months after acute infection in individuals with primary infection, while it took 12 months before the previously exposed group reached similar levels as in healthy controls. The majority of the effect came from the activated memory B cells expanding significantly more at 10 days after acute infection in the previously exposed group compared with primary infected individuals (1.7-fold increase, 95\% CI: 1.1-2.5, $P=0.0064)$. Both groups displayed a similar activated memory B cell decay rate, with a relatively long calculated half-life of 310 days (95\% CI: 259-387).

Resting memory B cells $\left(\mathrm{CD} 21^{+} \mathrm{CD} 27^{+}\right)$increased in overall cell frequency and numbers over time following infection (Figure $4 \mathrm{E}$ and Supplemental Figure 4D). These changes were not significantly different between the infected groups, although individuals with a primary infection displayed a more pronounced increase in the frequency of resting memory B cells compared with previously exposed individuals at 1 month after acute infection (Figure 4E). 
Naive B cell and CD27 $7^{\text {lo }}$ memory B cell $\left(\mathrm{CD} 21^{+} \mathrm{CD} 27^{-}\right)$frequencies were highly variable within infected groups and healthy controls. However, the average frequencies were similar over time, with the exception of a temporary increase in primary infected individuals and decrease in previously exposed individuals, at 10 days after acute infection (1.2-fold difference, 95\% CI: 1.0-1.4, $P=0.035$ ) (Figure 4F).

Atypical B cells $\left(\mathrm{CD} 21^{-} \mathrm{CD} 27^{-}\right)$rapidly expanded, both in individuals with a primary infection and in those with previous exposure at the acute time point compared with healthy controls (2.8-fold increase, 95\% CI: 1.6-4.9, $P=0.0002$ and 3.9-fold increase, 95\% CI: 2.3-6.4, $P<0.0001$, respectively) (Figure 4G). The atypical B cell frequencies then continued to increase and reached peak values at day 10, where the previously exposed group displayed a significantly higher frequency compared with individuals with primary infection (1.6-fold higher, 95\% CI: 1.1-2.5, $P=0.02$ ). After reaching peak levels, the atypical B cell compartment contracted slowly at a similar rate in both groups (Figure 4G). Primary infected individuals, with overall lower numbers of atypical B cells, reached background levels within 6 months after infection; whereas previously exposed individuals maintained a higher frequency at 12 months compared with controls. This was consistent with the overall levels of atypical B cells being higher in the previously exposed group (1.5-fold higher, 95\% CI: 1.1-2.1, $P=0.012$ ). The half-life for the atypical B cell compartment was estimated to 295 days ( $95 \%$ CI: $249-364$ ), suggesting that the cells were regulated similarly once generated and that they could be sustained for an extended period of time.

The mass cytometry data indicated that both activated memory B cells and atypical B cells expressed CD11c. We therefore assessed the kinetics of CD11 $\mathrm{c}^{+} \mathrm{B}$ cells following infection in both cohorts. Both malaria-infected groups displayed similar $\mathrm{CD} 11 \mathrm{c}^{+} \mathrm{B}$ cell dynamics as observed for activated memory and atypical B cells, which also resulted in a similar half-life estimate of 342 days (95\% CI: 279-442) (Figure 4H). The frequency of $\mathrm{CD} 11 \mathrm{c}^{+} \mathrm{B}$ cells was also highly correlated with the frequency of $\mathrm{CD} 21^{\text {lo }} \mathrm{B}$ cells $\left(\mathrm{r}^{2}=0.82, P<\right.$ 0.0001 ), indicating that CD11c was expressed by CD21 10 activated memory and atypical B cells (Figure $4 \mathrm{I}$ ). To assess to what extent expression of CD11c could represent recently activated cells, we measured the intracellular proliferation marker Ki67 in mature B cells from 26 individuals (10 primary infected and 16 previously exposed) at the acute time point. We found that $78 \%$ of $\mathrm{Ki}^{+}$mature B cells also expressed CD11c (Figure $4 \mathrm{~J})$, strongly supporting expression of CD11c as a marker of recently activated B cells during malaria. There was no difference between the primary infected and previously exposed groups $(P=0.18$, Mann-Whitney test), indicating a similar upregulation of CD11c in responding cells of both groups at the acute time point.

$C D 11 c^{+} B$ cells are composed of several $B$ cells subsets. Since $C D 11 c^{+} B$ cells expanded differently depending on previous exposure to malaria parasites, we wanted to further understand the composition of this compartment and determine if there were qualitative changes over time following infection. To do this, we first gated on $\mathrm{CD} 19^{+} \mathrm{CD} 20^{+} \mathrm{CD} 10^{-}$mature $\mathrm{B}$ cells followed by $\mathrm{CD} 11 \mathrm{c}$. We then com-

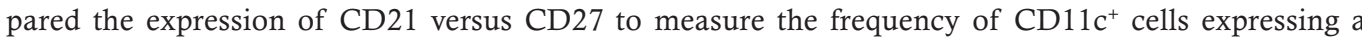
naive $\left(\mathrm{CD} 21^{+} \mathrm{CD} 27^{-}\right)$, resting memory $\left(\mathrm{CD} 21^{+} \mathrm{CD} 27^{+}\right)$, activated memory $\left(\mathrm{CD} 21^{-} \mathrm{CD} 27^{+}\right)$, and atypical $\left(\mathrm{CD} 21^{-} \mathrm{CD} 27^{-}\right) \mathrm{B}$ cell phenotype at each time point (Figure 5A). Interestingly, we found that CD11c was expressed by several B cell subsets, with atypical B cells and resting memory B cells constituting the majority of cells (Figure 5B). Overall, the frequency of the cell populations was very similar between the groups. However, the distribution of $\mathrm{B}$ cell subsets within the $\mathrm{CD} 11 \mathrm{c}^{+}$compartment was highly dynamic, with a rapid expansion of activated and atypical B cells coinciding with a reduction in the frequency of resting memory B cells (Figure 5B). To understand if the dynamic pattern was due to an expansion or contraction of specific cell subsets, we normalized cell numbers to gated live lymphocytes for each donor (Figure 5C). Previously exposed individuals had a larger number of cells within each $\mathrm{B}$ cell subset, consistent with them having overall more $\mathrm{CD} 11 \mathrm{c}^{+} \mathrm{B}$ cells. However, we only observed a clear expansion in atypical and activated memory B cell subsets, indicating that the remodeling within the $\mathrm{CD} 11 \mathrm{c}^{+} \mathrm{B}$ cell compartment was primarily due to expansion of cells, rather than a redistribution.

Selective expansion of switched $C D 11 c^{+} B$ cells in previously exposed individuals followed $P$. falciparum infection. To further investigate the effect of de novo versus memory responses, we separated $\mathrm{CD} 11 \mathrm{c}^{+} \mathrm{B}$ cells based on expression of IgD. In the mass cytometry experiment, we found that individuals with primary infection $(n=2)$ had more $\operatorname{IgD}^{+} \mathrm{CD}_{11} \mathrm{c}^{+}$cells compared with previously exposed individuals $(n=2)$ (Figure 6A). To confirm this observation in the full sample cohort $(n=49)$, we also measured the frequency

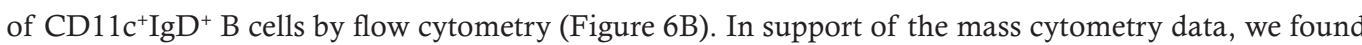
that the overall frequency of $\mathrm{CD} 11 \mathrm{c}^{+} \mathrm{IgD}^{+} \mathrm{B}$ cells was significantly higher for individuals with a primary infection compared with those with previous malaria exposure (1.4-fold increase, 95\% CI: 1.1-1.8, $P=$ 
A
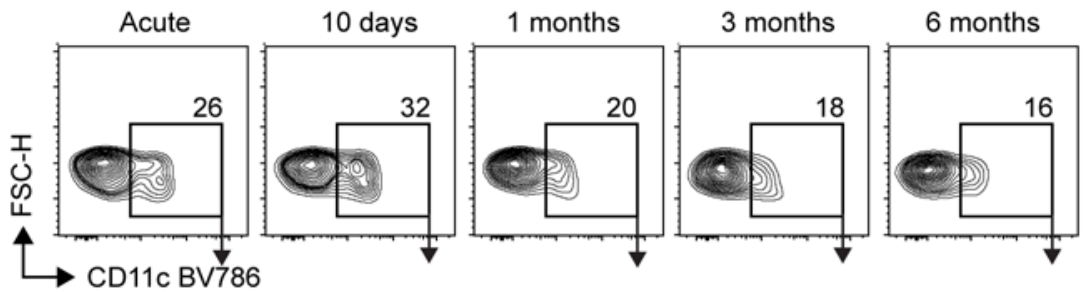

12 months
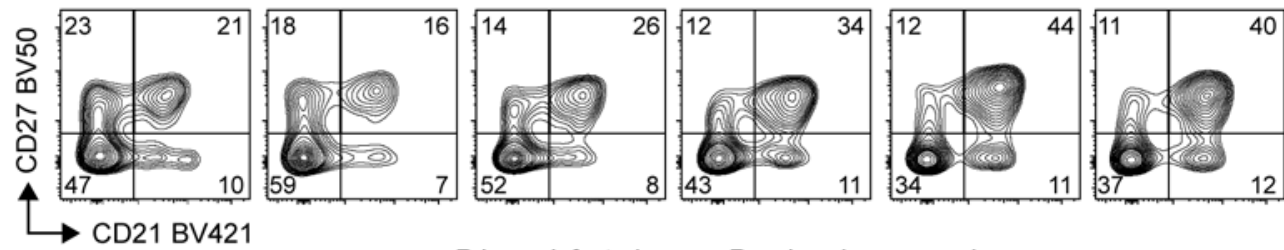

B Activated memory
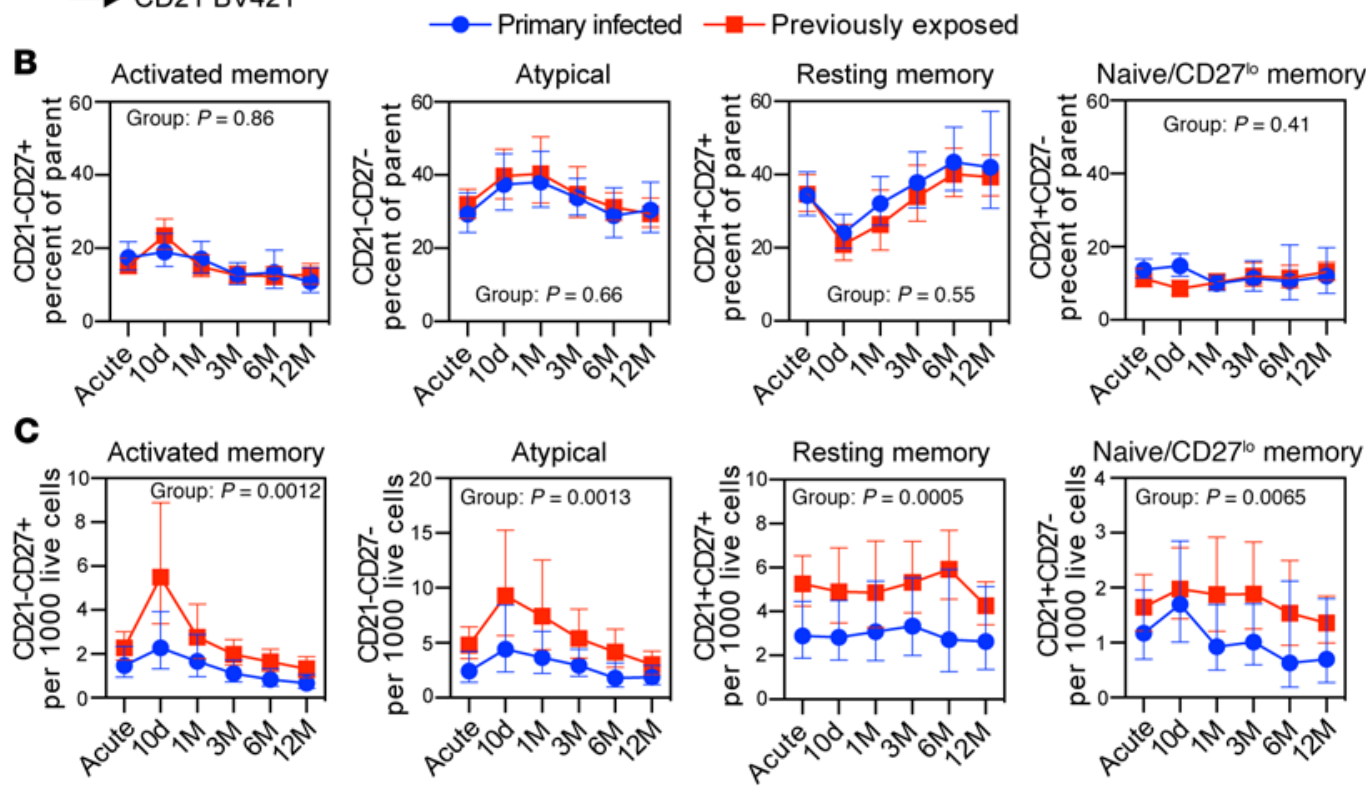

Figure 5. CD11c B cell subset kinetics in primary infected and previously exposed individuals. (A) Schematic gating strategy for $\mathrm{CD}_{11 \mathrm{C}^{+}} \mathrm{B}$ cells followed by CD21 versus CD27 in one previously exposed donor at the acute, 10-day and 1-, 3-, 6-, and 12-month time points. (B) Frequency of CD11 $\mathrm{C}^{+} \mathrm{B}$ cells further separated based on expression of CD21 and CD27. (C) Cell numbers per thousand live lymphocyte singlets for CD11 $\mathrm{C}^{+} \mathrm{B}$ cells indicated in $\mathbf{A}$. Statistical differences between groups were assessed using a linear mixed-effects model, with the $P$ value indicated in the graphs. Primary infected individuals are indicated by blue circles $(n=16)$, and previously exposed individuals by red boxes $(n=33)$. Symbols indicate geometric mean with error bars showing $\pm 95 \% \mathrm{Cl}$.

0.0049). We then separated the individual $\mathrm{CD} 11 \mathrm{c}^{+} \mathrm{B}$ cell subsets based on IgD expression and normalized to gated live lymphocytes (Figure 6C). The numbers and dynamics of $\mathrm{IgD}^{+} \mathrm{B}$ cells in each subset were highly similar between primary infected and previously exposed individuals (group comparisons, $P>0.05$ ), suggesting that these cells likely represented de novo responses in both groups (Figure 6D). All $\mathrm{IgD}^{+} \mathrm{B}$ cell subsets displayed a dynamic response, characterized by an extensive expansion at 10 days after the acute time point, followed by contraction of the compartments (time effect, $P<0.05$ ). In contrast, the number of $\mathrm{IgD}^{-} \mathrm{B}$ cells was significantly higher in all $\mathrm{CD} 11 \mathrm{c}^{+} \mathrm{B}$ cell subsets in previously exposed individuals (group comparison, $P<0.05$ ) (Figure 6E). However, only atypical and activated memory B cells showed a dynamic response to the infection (time effect, $P<0.05$ ), suggesting that these $B$ cell subsets were generated from reactivated memory B cells.

Differential marker expression over time in $C D 11 c^{+} B$ cells. To further assess the phenotype of $\mathrm{CD} 11 \mathrm{c}^{+} \mathrm{B}$ cells, we compared the expression profile of $\mathrm{CD} 11 \mathrm{c}^{+}$and $\mathrm{CD} 11 \mathrm{c}^{-} \mathrm{B}$ cells to CD20, CD19, CD21, CD85j, CXCR3, and FcRL5 (Figure 7A). CD11 $\mathrm{c}^{+} \mathrm{B}$ cells expressed higher levels of CD19, CD20, CD85j, CXCR3, and FCRL5 (ratio > 1) and lower levels of CD21 (ratio < 1) compared with CD11 $\mathrm{c}^{-} \mathrm{B}$ cells (Figure 7B). FcRL5 and CXCR3 were highly expressed by CD11 $\mathrm{c}^{+} \mathrm{B}$ cells; however, not all cells expressed 
A

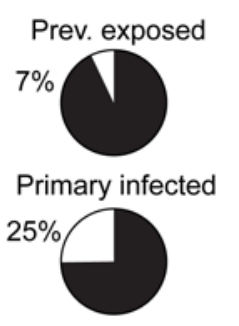

D $\lg \mathrm{D}^{+}$

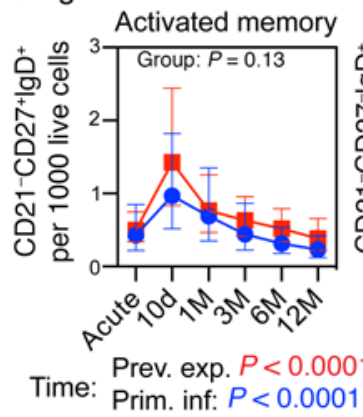

B

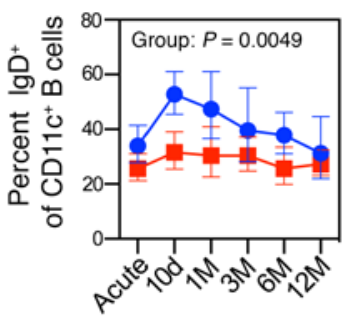

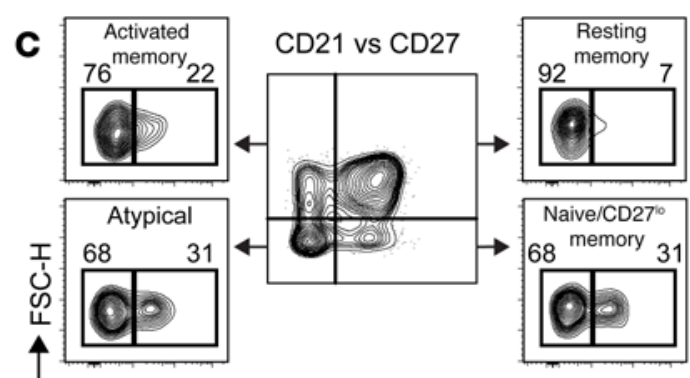

IgD FITC

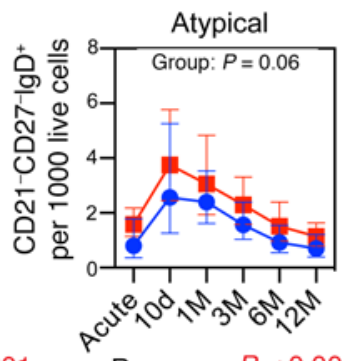

Prev. exp. $P<0.000$ Prim. inf. $P<0.0001$

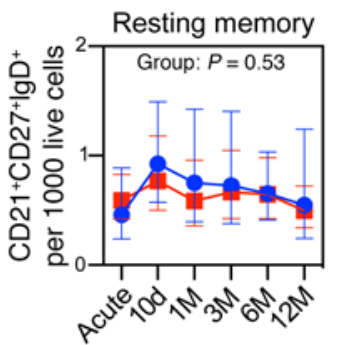

Prev. exp. $P=0.22$ Prim. inf. $P=0.04$

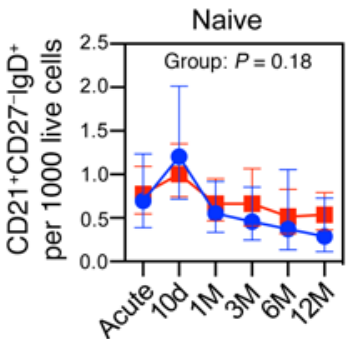

Prev. exp. $P=0.008$ Prim. inf. $P<0.0001$

$\mathbf{E}_{\text {IgD- }}$
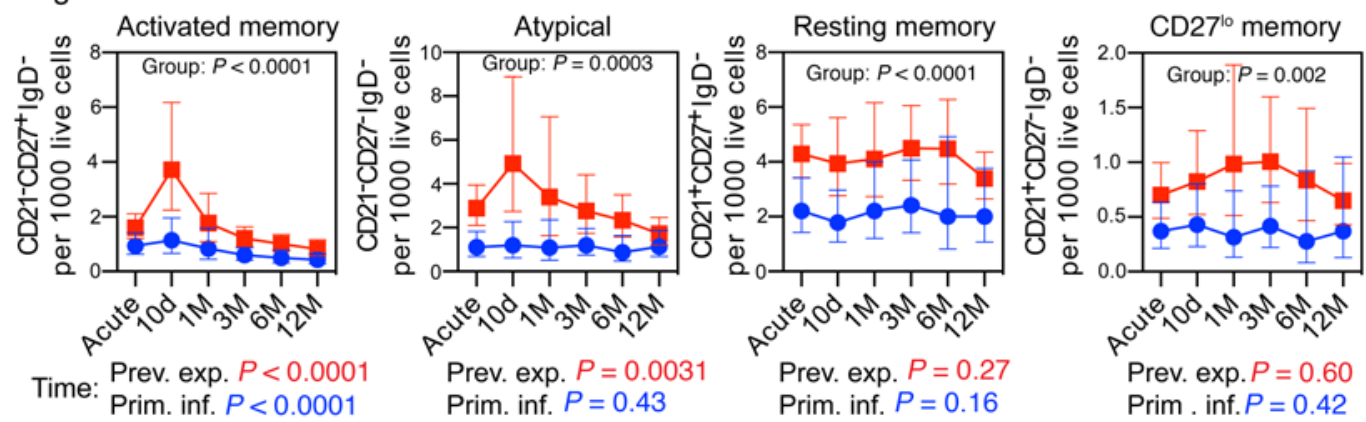

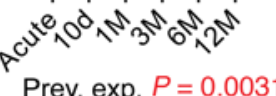

Prev. exp. $P=0.0031$ Prim. inf. $P=0.43$

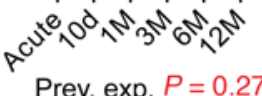

Prim. inf. $P=0.16$
60

Prev. exp. $P=0.60$

Prim . inf. $P=0.42$

Figure 6. Expansion of switched CD11c $B$ cells is associated with previous exposure to $P$. falciparum parasites. (A) Frequency of IgD+CD11 ${ }^{+} B$ cells in primary infected $(n=2)$ and previously exposed $(n=2)$ individuals, as determined by mass cytometry. (B) Frequency of IgD+ cells among CD11c $\mathrm{B}$ cells, as determined by flow cytometry. (C) Schematic gating strategy for assessing IgD expression in CD11 ${ }^{+} \mathrm{B}$ cell subsets separated by expression of CD21 and CD27. (B, D, and E) Cell numbers per thousand live lymphocyte singlets for (D) IgD+ or (E) IgD- CD11c ${ }^{+}$B cell subsets. Statistical differences between groups (B, D, and $\mathbf{E}$ ) and in the B cell subset dynamics over time ( $\mathbf{D}$ and $\mathbf{E}$ ) were assessed using a linear mixed-effects model and are indicated within and underneath the graphs by group and time, respectively. The same color scheme and donor numbers $(n)$ are used throughout the figure (B, $\mathbf{D}$, and $\mathbf{E}$ ), with primary infected individuals indicated by blue circles/text $(n=16)$ and previously exposed individuals by red boxes/text $(n=33)$. Lines indicate geometric mean $\pm 95 \% \mathrm{Cl}$.

the markers (Figure 7A). To determine if this was due to dynamic expression over time, or attributed to differences between the groups, we measured the frequency of CD11 $\mathrm{c}^{+} \mathrm{B}$ cells that also expressed FcRL5 and CXCR3 over time. We found that FCRL5 was expressed in approximately $70 \%$ of CD $11 \mathrm{c}^{+} \mathrm{B}$ cells at the time of acute infection (Figure 7C). The proportion of FCRL $5^{+}$cells then decreased slightly over time to reach approximately $50 \%$ at 12 months after infection, remaining slightly above the frequency found in $\mathrm{CD} 11 \mathrm{c}^{+} \mathrm{B}$ cells of healthy controls. To assess if the decline was due to a redistribution of $\mathrm{CD} 11 \mathrm{c}^{+} \mathrm{B}$ cell subsets, we separated the cells based on expression of CD21 and CD27 as before. We found that FcRL5 was expressed in almost all $\mathrm{CD} 21^{-} \mathrm{CD} 27^{+}$activated memory B cells and $\mathrm{CD} 21^{-} \mathrm{CD} 27^{-}$atypical $\mathrm{B}$ cells (Figure 7D). However, in $\mathrm{CD} 21^{+} \mathrm{CD} 27^{+}$resting memory B cells and $\mathrm{CD} 21^{+} \mathrm{CD} 27^{-}$naive $/ \mathrm{CD} 27^{10}$ memory cells, the expression of FcRL5 was only temporary and rapidly contracted following 10 days after acute infection (Figure 7D). CXCR3 was slower to be expressed and peaked at approximately $60 \%$ of $\mathrm{CD} 11 \mathrm{c}^{+} \mathrm{B}$ cells at 10 days to 1 month after acute infection (Figure $7 \mathrm{E}$ ). The frequencies then slightly declined over 3 months after infection, after which the levels remained stable. In contrast to FcRL5, 
CXCR3 displayed a similar kinetics in all CD11 $\mathrm{c}^{+} \mathrm{B}$ cells subsets, although the frequency of cells expressing the marker varied considerably (Figure 7F). The frequency of CXCR3 ${ }^{+}$cells was highest among $\mathrm{CD} 21^{-} \mathrm{CD} 27^{+}$activated memory B cells, with approximately $80 \%$ of the cells expressing the maker. This was followed by CD21-CD27- atypical B cells, with approximately $50 \%$ of cells expressing CXCR3 followed by $\mathrm{CD} 21^{+} \mathrm{CD} 27^{+}$resting memory B cells and $\mathrm{CD} 21^{+} \mathrm{CD} 27^{-}$naive/CD27 lo memory cells, with approximately $30 \%-40 \%$ of cells expressing CXCR3.

T-bet is suggested as a key driver in the formation of atypical B cells in mice (38) and humans (15). To assess the expression of T-bet among CD11 ${ }^{+} \mathrm{B}$ cells, we measured intracellular T-bet by flow cytometry at the time of acute infection in a subset of patients (primary infected, $n=10$; previously exposed, $n=16$ ). Among mature B cells, we found that T-bet was expressed in $33 \%$ of CD11 $\mathrm{c}^{+} \mathrm{B}$ cells, while only $1.1 \%$ of CD11 ${ }^{-}$B cells expressed T-bet $(P<0.0001, n=26)$ (Supplemental Figure 5A). Furthermore, among

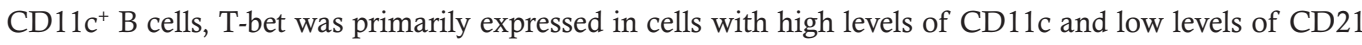
with variable levels of CD27 (Supplemental Figure 5B), indicating that high expression of T-bet is associated with atypical $\mathrm{B}$ cells and activated memory B cells. T-bet ${ }^{-} \mathrm{CD} 11 \mathrm{c}^{+} \mathrm{B}$ cells primarily expressed $\mathrm{CD} 27$, suggesting an enrichment for activated and resting memory B cells.

$C D 11 c^{+} B$ cells are enriched for $P$. falciparum-specific B cell clones. As CD11c was expressed in B cells expanding during malaria, we wanted to evaluate if the responding cells were specific for $P$. falciparum-specific B cell clones. We sorted switched CD11 $\mathrm{c}^{+}$and CD11 $\mathrm{c}^{-} \mathrm{B}$ cells at 2 cells per well into 384-well culture plates supplemented with IL-2, IL-21, and feeder cells expressing CD40 ligand. The B cells were sorted from 3 previously exposed donors ( $n=4606$ wells) at 10 days after acute infection and 2 healthy controls ( $n=1000$ wells) (Table 1 and Supplemental Figure 6). Following 2 weeks of incubation, we assessed IgG production by ELISA. CD11c ${ }^{+}$B cells differentiated to antibody-secreting cells at a slightly lower rate compared with $\mathrm{CD} 11 \mathrm{c}^{-} \mathrm{B}$ cells (Table 1 and Supplemental Figure 6), a finding that can be potentially attributed to a larger contribution from atypical B cells among $\mathrm{CD} 11 \mathrm{c}^{+}$cells. To further determine if the produced antibodies were malaria specific, IgG from high producing wells (OD > 0.2-0.3 in IgG ELISA) were screened for binding to plasmodum falciparum schizont

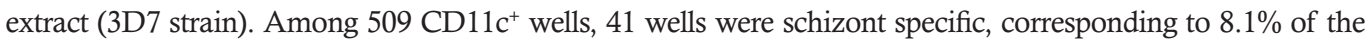
responding wells. In comparison, among $566 \mathrm{CD} 11 \mathrm{c}^{-}$wells, 15 were schizont specific, corresponding to $2.7 \%$ of the responding wells (Table 1). Thus, schizont-specific B cell clones were significantly enriched in CD11c ${ }^{+} \mathrm{B}$ cells compared with CD11 $\mathrm{c}^{-} \mathrm{B}$ cells (3-fold, range 2.2-4.4-fold, $P<0.0001$, Fisher's exact test).

Since atypical B cells are known to also be enriched for self-reactive or polyreactive B cell clones (19) and constitute a large portion of the $\mathrm{CD} 11 \mathrm{c}^{+} \mathrm{B}$ cells, it is possible that antibodies from these cells could bind nonspecifically to schizont extracts. To determine if this was the case, we screened $500 \mathrm{CD}_{11 \mathrm{c}^{+} \text {wells }}$ from 2 healthy controls for binding to schizont extracts. From 174 out of 500 wells, producing high levels of IgG, none showed binding to schizont extract, clearly indicating that binding to schizonts was not due to polyreactivity, but rather indicative of parasite-specific B cell clones.

\section{Discussion}

In this study, the primary and secondary B cell response to infection with $P$. falciparum malaria was investigated in a cohort of travelers followed over 1 year after infection. We believe this study enables a robust characterization of B cell dynamics and the contribution of memory to the B cell response after natural infection with $P$. falciparum in the absence of parasite reexposure. Our results revealed a strong activation of the B cell compartment associated with an expanded population of B cells with high expression of CD11c and low expression of CD21. This expansion was also significantly stronger in individuals with previous $P$. falciparum exposure, compared with those with a primary infection, suggesting activation of preexisting parasite-specific memory B cells in previously exposed individuals. This was corroborated by an abundant production of parasite-specific antibodies in the group with previous exposure.

We found that approximately $80 \%$ of all mature B cells that proliferated in response to acute infection also expressed CD11c. To determine if this was due to polyclonal activation of the B cell compartment, as has been described to occur during malaria (39), or an antigen-specific response, we assessed the binding specificity of B cells with or without surface expression of CD11c. However, the $P$. falciparum genome contains $>5000$ genes (40). It is therefore difficult to accurately assess the complete parasite-specific B cell repertoire. Schizont extract consists of lysed red blood cells infected with whole parasites, which present many of the available blood-stage antigens. By screening with schizont extract, we could therefore sample a wider B cell repertoire compared with the use of single purified antigens. We found that $8.1 \%$ of $C D 11 c^{+}$ 

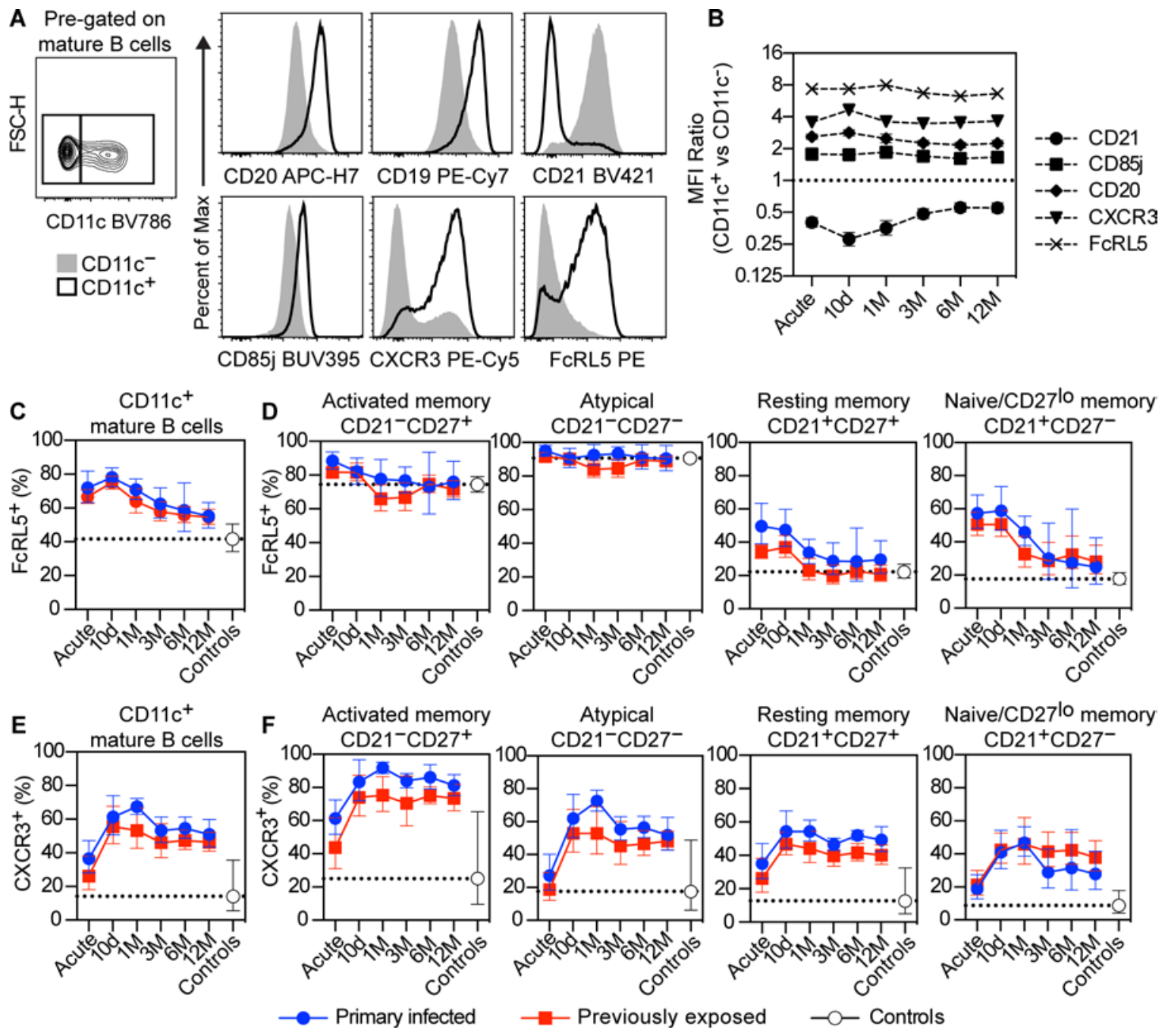

Figure 7. Dynamic expression of FcRL5 and CXCR3 among CD11c ${ }^{+} B$ cell subsets. (A) Representative graph showing gating of CD11c ${ }^{+}$and $C D 11 c^{-}$mature $B$ cells (CD19+CD20+CD10') from 1 previously exposed donor at 10 days after acute infection (left). Representative expression levels of CD20, CD19, CD21, CD85j,

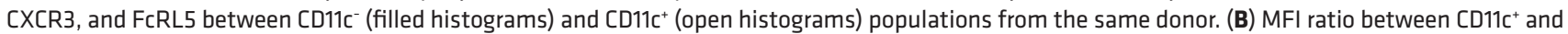
CD11 $c^{-}$mature B cells for CD21 (circles), CD85j (boxes), CD20 (diamonds), CXCR3 (triangles), and FcRL5 (crosses) at each sample time point. Data represent both primary infected and previously exposed individuals $(n=49)$. (C) Frequency of FCRL $5^{+}$cells among total CD11c ${ }^{+}$mature B cells or $(D)$ further separated based on expression of CD21 and CD27. (E) Frequency of CXCR3 $3^{+}$cells among total CD11 ${ }^{+}$mature B cells or (F) further separated based on expression of CD21 and CD27. (C-F) Data show geometric mean with error bars indicating $\pm 95 \% \mathrm{Cl}$. Primary infected individuals are indicated by blue circles $(n=16)$, previously exposed individuals by red boxes $(n=33)$, and healthy controls by white circles $(n=14)$. The dotted line indicates the geometric mean of the healthy controls.

B cells were specific for $P$. falciparum schizonts in previously exposed individuals, corresponding to a 2- to 4-fold enrichment compared with CD11 $\mathrm{c}^{-}$B cells. This supports CD11c as a selective marker for B cells responding to parasites during an episode of malaria.

The CD11 $\mathrm{c}^{+} \mathrm{B}$ cells were significantly expanded in infected individuals compared with healthy controls at the acute infection, indicating that these cells were rapidly generated following infection. The cells expanded further until 10 days after acute infection, where after they started to contract. The contraction was similar between the groups, with a half-life estimate of approximately 300 days. This suggests that, once generated, the cells were regulated similarly, independent of origin.

To assess the B cell subsets making up the CD11c $\mathrm{c}^{+} \mathrm{B}$ cell compartment further, we separated the cells based on expression of CD21 and CD27. Interestingly, we found that both atypical B cells and resting memory B cells each contributed approximately $30 \%-40 \%$ of the CD $11 \mathrm{c}^{+}$compartment, indicating that CD $11 \mathrm{c}$ expression is not limited to atypical B cells, but rather a marker for peripheral B cell subsets responding during malaria. We also found that the $\mathrm{B}$ cell subset distribution was highly dynamic over time, indicating an active remodeling of the $\mathrm{CD} 11 \mathrm{c}^{+}$compartment in response to the infection. Both infected cohorts dis- 
Table 1. Schizont-specific clones are enriched in the CD11c ${ }^{+}$B cell compartment

\begin{tabular}{|c|c|c|c|c|c|c|}
\hline Groups & Donors (n) & Sorted wells $(n)$ & $\operatorname{lgG}^{+}(\%)$ & Screened for Ag binding ( $n$ ) & Schizont $^{+}(\%)$ & Statistics \\
\hline Previously exposed & 3 & 4606 & $1442(31.3)$ & 1075 & $56(5.2)$ & $P=0.0003^{A}$ \\
\hline $\mathrm{CD} 11 \mathrm{c}^{+}$ & & 2306 & $616(26.7)$ & 509 & $41(8.1)$ & $P<0.0001^{\mathrm{B}}$ \\
\hline Healthy controls & 2 & 1000 & $265(26.5)$ & 174 & 0 & \\
\hline $\mathrm{CD}_{11 \mathrm{c}^{+}}$ & & 500 & $157(31.4)$ & 106 & 0 & \\
\hline $\mathrm{CD} 11 \mathrm{c}^{-}$ & & 500 & $108(21.6)$ & 68 & 0 & \\
\hline
\end{tabular}

${ }^{A}$ Comparison of total schizont ${ }^{+}$wells between previously exposed individuals and healthy controls using Fisher's exact test. ${ }^{B}$ Comparison of CD11c ${ }^{+}$and CD11c $^{-}$schizont ${ }^{+}$wells within previously exposed individuals using Fisher's exact test.

played a similar expansion of unswitched cells, suggesting that this corresponded to a de novo response. In contrast, the expansion of switched cells was only observed in previously exposed individuals, suggesting that these cells were derived from preexisting antigen-specific memory B cells.

These results are consistent with clonal lineage analysis of $\mathrm{B}$ cells, which has demonstrated that atypical $\mathrm{B}$ cell $\mathrm{BCR}$ characteristics and replication history closely resemble that of resting memory $\mathrm{B}$ cells in both mice (41) and humans $(11,42)$. For unswitched atypical B cells, there are less data available to suggest their potential origin. Bagnara et al. showed that unswitched $\mathrm{CD} 27^{+}$memory B cells shared antibody sequence characteristics with unswitched $\operatorname{IgM}^{+} \mathrm{CD} 21^{-} \mathrm{CD} 27^{-}$atypical B cells, indicating a potential relationship (43). Both naive and memory $\mathrm{B}$ cells were shown to express $\mathrm{T}$-bet, associated with $\mathrm{CD} 11 \mathrm{c}$ expression, in vitro when stimulated with recombinant IFN- $\gamma$ and anti-Ig (15). As $P$. falciparum infection is known to induce a strong IFN- $\gamma$ response (44), this could explain how mature B cells, including resting memory and naive cells, also upregulate $\mathrm{CD} 11 \mathrm{c}$. That both naive and memory $\mathrm{B}$ cells can be made to express T-bet in vitro is interesting, as up to half of the atypical B cells express an unswitched BCR in malaria-endemic areas $(11,18)$. This suggests that recently activated unswitched B cells could favor differentiation to atypical B cells. Obeng-Adjei et al. further showed that stimulation of light-zone GC B cells with recombinant IFN- $\gamma$ and BCR ligation would lead to upregulation of T-bet in vitro (15). This is consistent with the findings of Lau et al., which propose that $\mathrm{CD} 21^{-} \mathrm{CD} 27^{+}$activated memory B cells, which we here show express CD11c and are strongly expanded in peripheral blood during $P$. falciparum infection, correspond to cells that recently emigrated from the GC (45).

It is unclear if direct differentiation between $\mathrm{CD} 11 \mathrm{c}^{+} \mathrm{B}$ cell subsets occurs in peripheral blood, as naive and memory $B$ cell activation and differentiation generally occur in secondary lymphoid organs (46). $C D 11 \mathrm{c}^{+} \mathrm{B}$ cells presenting with reduced BCR responsiveness and high expression of CD20 and CD95 and low expression of CD21 can be found in tonsils of healthy individuals (47, 48). However, few of these cells enter peripheral blood during steady state, suggesting that malaria somehow perturbs the normal regulation of these cells. It will be important to elucidate this dysregulation, but also to further determine the origin and regulation of the peripheral $\mathrm{CD} 11 \mathrm{c}^{+} \mathrm{B}$ cell subsets, to fully understand the effect of malaria on the immune response.

Here, we investigated B cell responses in a potentially unique study cohort following natural infection with $P$. falciparum over time. Using an explorative approach with mass cytometry, followed by targeted flow cytometry, we could clearly demonstrate that $\mathrm{CD} 11 \mathrm{c}$ constitutes a selective marker for B cells that respond to P. falciparum infection. We could also show that $\mathrm{CD} 11 \mathrm{c}$ was upregulated by several $\mathrm{B}$ cell subsets upon infection. It will be important to further study the role and regulation of these subsets during infection. By characterizing the $\mathrm{CD} 11 \mathrm{c}^{+} \mathrm{B}$ cells longitudinally, we found that primary infected and previously exposed individuals displayed a similar expansion of unswitched $\mathrm{CD} 11 \mathrm{c}^{+} \mathrm{B}$ cells, indicating a de novo response to the infection. In contrast, individuals with previous $P$. falciparum exposure had an increased expansion of switched $\mathrm{CD} 11 \mathrm{c}^{+}$atypical and activated memory $\mathrm{B}$ cells, absent during primary infection. We could also show that the $\mathrm{CD} 11 \mathrm{c}^{+} \mathrm{B}$ cell compartment had a long half-life, which could explain how high levels of atypical B cells can be maintained in individuals living in malaria-endemic areas. Taken together, our data offer an improved understanding of how primary and secondary malaria-specific B cell responses expand and contract following acute malaria. These findings can contribute in the design and interpretation of future studies aiming to develop B cell-specific intervention strategies against malaria. 


\section{Methods}

Study design. A prospective study enrolling adult patients diagnosed with $P$. falciparum malaria was performed at Karolinska University Hospital in Stockholm, Sweden, from 2011 to 2017. Fifty-one adult patients treated for $P$. falciparum infection and fourteen healthy controls were included. Study participants were grouped according to previous malaria exposure and origin. This enabled comparison of immune responses in malaria-naive individuals of primarily Swedish origin, who contracted malaria for the first time ( $n=17$, called primary infected), with individuals originating from malaria-endemic areas in Sub-Saharan Africa $(n=34$, called previously exposed). Clinical data were extracted from medical records, and a questionnaire relating to the participant health status, previous traveling, and malaria exposure was filled in by all participants.

Sampling scheme. Patients were invited for sampling at the time of malaria diagnosis and then at 10 days, 1 month, 3 months, 6 months, and 12 months (Figure 1 and Supplemental Figure 7). Fourteen healthy volunteers who never travelled to malaria-endemic areas were recruited as controls and sampled at 1 time point. On each sampling occasion, $36 \mathrm{ml}$ venous blood was collected using sodium heparin or EDTA tubes. PBMCs were isolated using Ficoll-Paque density gradient separation, resuspended in $90 \%$ fetal calf serum supplemented with $10 \% \mathrm{DMSO}$, and stored at $-150^{\circ} \mathrm{C}$. In addition to isolation and freezing of PBMCs at each follow-up, samples were also collected for antibody analysis (serum or plasma), blood chemistry and microscopy, and PCR and blood smears for detection of malaria parasites. Part of the cohort has been described previously for telomere dynamics (49) and parasite prevalence after treatment (50). For some time points and study participants, only plasma or cells were available, explaining the different $(n)$ included in the analysis. No samples were excluded if data were available.

Detection of $P$. falciparum parasites. Parasites were detected and enumerated by light microscopy of Fields stained thick and thin blood smears at the Department of Clinical Microbiology at Karolinska University Hospital in addition to PCR as described previously (51).

Leukocyte phenotyping. White blood cell differential counts were performed using the Sysmex XS-1000i platform, Sysmex Corporation, at the Department of Clinical Chemistry at Karolinska University Hospital.

Mass cytometry (CyTOF). Phenotypic analysis of B cells via mass cytometry was performed on sample series from 4 infected individuals. Two previously exposed individuals ( $n=11$ samples), two primary infected individuals ( $n=10$ samples), and two controls ( $n=3$ samples) were included (see Figure 2). Approximately 2 million PBMCs were thawed from each sample, stained, and subsequently analyzed by CyTOF (Fluidigm Inc.) using a predefined 29-marker panel targeting predominantly B cell-associated cell surface molecules (Supplemental Table 2). Samples were prepared as described in the Supplemental Methods. Briefly, cells were thawed, washed, and rested overnight at $37^{\circ} \mathrm{C}$ with $5 \% \mathrm{CO}_{2}$ before staining with the viability marker cisplatin, followed by surface markers and intracellular staining for Ki-67 on day 2. On day 3, a DNA stain was added and the samples analyzed by CyTOF.

Analysis of mass cytometry data. The resulting data files were gated for live CD $45^{+} \mathrm{CD} 3^{-} \mathrm{CD} 19^{+} \mathrm{B}$ cells using FlowJo X version 10.4.2 Exported B cells were then analyzed using the Cytofkit version 1.12.0, which includes an integrated pipeline for mass cytometry analysis (52). Prior to analysis, all samples were normalized to equal cell numbers. We then used tSNE to reduce dimensionality of the data followed by Phenograph for clustering of the data into subpopulations. The resulting subpopulations were further analyzed for specific marker expression and frequency at the different time points after infection and in each individual.

Flow cytometry. Frozen PBMCs were thawed in a $37^{\circ} \mathrm{C}$ water bath and mixed with 1 equal volume cold Iscove's Modified Dulbecco's Medium supplemented with L-glutamine (2 mM), penicillin (100 U/ml), streptomycin $(100 \mu \mathrm{g} / \mathrm{ml})$, and $10 \%$ heat-inactivated fetal bovine serum (all from Thermo Fischer Scientific). Cells were then rested 20 minutes on ice before being washed twice in DPBS lacking magnesium or calcium. After washing, the cells were incubated with Aqua Live/Dead stain (Thermo Fisher Scientific) for 20 minutes followed by further washing in DPBS supplemented with $2 \%$ FBS. The cells were then incubated for 20 minutes on ice, in 2 steps with 2 washes in between, using an antibody mix targeting primarily B cell surface antigens (Supplemental Table 3). After staining, the cells were washed twice in DPBS with 2\% FBS before acquisition on a 5-laser BD LSRFortessa flow cytometer. Gating was done with FlowJo X software version 10.4.2, with the gating strategy shown in Supplemental Figure 3.

For intracellular staining of T-bet and Ki67, the cells were first stained for surface makers as described above. The cells were then fixed and permeabilized using the FoxP3 buffer staining kit for 30 minutes at room temperature (Thermo Fisher Scientific). After washing, the cells were stained with the antibodies for 30 minutes at room temperature followed by washing and acquisition. 
Low-density B cell culture. The B cell culture assay was performed as described previously (53), with some modifications. Briefly, PBMCs were thawed and stained with markers enabling sorting of IgGswitched CD11 $\mathrm{c}^{+}$and CD11 $\mathrm{c}^{-}$B cells (see Supplemental Table 4 for markers). The same staining protocol as described in the Flow cytometry section was used. Cells were sorted on a 5-laser BD Influx at a density of 2 cells per well into a 384 -well plate containing $50 \mu 1$ glutamax minimal essential media supplemented with $10 \%$ heat-inactivated fetal bovine serum, $100 \mathrm{U} / \mathrm{ml}$ penicillin, $100 \mu \mathrm{g} / \mathrm{ml}$ streptomycin, and $2 \mathrm{mM}$ glutamine (all from Thermo Fisher Scientific). Each well further contained $10 \mathrm{ng} / \mathrm{ml} \mathrm{IL-2}$ and $10 \mathrm{ng} / \mathrm{ml} \mathrm{IL}-21$ (both from Peprotech) and 5,000 3T3-msCD40L feeder cells (provided by John Mascola and Nicole DoriaRose of Vaccine Research Center, NIH, Bethesda, MD, USA) that were previously irradiated with 50 Gy. Following sorting the plates were placed at $37^{\circ} \mathrm{C}$ supplemented with $5 \% \mathrm{CO}_{2}$ and incubated for $10-16$ days

Screening of B cell cultures. B cell culture supernatants were screened for total IgG production by ELISA. Nunc 384-well plates were coated with $1 \mu \mathrm{g} / \mathrm{ml}$ goat anti-human IgG/IgM polyclonal antibody (Jackson Immunoresearch) followed by blocking with $1 \%$ bovine serum albumin (BSA, MilliporeSigma) in PBS. Five $\mu \mathrm{l}$ culture supernatant was then added to wells containing $20 \mu 1$ PBS with $0.1 \%$ BSA followed by detection with goat anti-human IgG (Jackson Immunoresearch) conjugated to HRP diluted 1:10,000 in PBS with $0.1 \%$ BSA. The signal was developed with 3,3',5,5'-Tetramethylbenzidine (TMB) and stopped with $1 \mathrm{M} \mathrm{H}_{2} \mathrm{SO}_{4}$. OD was read at $450 \mathrm{~nm}$ using a Victor Wallac 1420 (Perkin Elmer).

Screening for malaria-specific IgG was done as for total IgG, with the difference that 384-well ELISA plates were coated with schizont extract (3D7 clone) at a concentration of $2 \mu \mathrm{g} / \mathrm{ml}$ instead.

Schizont IgG ELISA. Malaria-specific IgG antibodies were measured in plasma using a schizont extract ELISA according to a previously described protocol (54). Briefly, Nunc MaxiSorp flat-bottom plates (Nunc) were coated with P. falciparum schizont extract (3D7 clone, provided by Mats Wahlgren, Karolinska Institutet, Stockholm, Sweden) at a concentration of $2 \mu \mathrm{g} / \mathrm{ml}$. Plasma samples were analyzed at 1:1000 dilution. Bound malaria-specific IgG was detected by HRP-conjugated rabbit anti-human IgG (Dako). Plates were developed using o-phenylenediamine dihydrochloride, and ODs were read at 492 $\mathrm{nm}$ using a Vmx Kinetic microplate reader (Molecular Devices).

$B$ cell subset half-life calculation. Linear mixed-effects models were used to examine the rate of contraction within the $\mathrm{CD} 21^{-} \mathrm{CD} 27^{+}$activated memory, the $\mathrm{CD} 21^{-} \mathrm{CD} 27^{-}$atypical, and the $\mathrm{CD} 11 \mathrm{c}^{+}$compartments after infection. For the purpose of this analysis, the data set was curated to only include data from the contraction phase of the response. The models were fitted individually to $\log _{\mathrm{e}}$-transformed data on cell frequencies for each cell subset, allowing for both an individual initial cell number (random intercept) and rate of contraction (random slope). The estimated average rate of contraction within each of the compartments was expressed as the compartment half-life (calculated as $\log _{e}(2) /$ slope). There was no significant difference in the rate of contraction between the 2 exposure groups ( $\triangle \mathrm{AIC}>10$ for alternative model including interaction between prior exposure and time).

Statistics. Statistical analyses were performed using SAS JMP (version 14.0.0), GraphPad Prism (version 6) and R (version 3.4.1). Prior to analysis data were $\log _{\mathrm{e}}$ transformed. All statistical tests were 2 tailed. A $P$ value $<0.05$ was considered significant. Comparisons between controls and infected individuals at selected time points were done using ANOVA followed by Tukey's HSD test, which controls for repeated measures. Comparisons between groups were presented as fold difference with a $95 \%$ CI. Comparisons of variables within the same individual were evaluated using Wilcoxon's matched pairs test and between infected individuals with the Mann-Whitney $U$ test. Overall differences in group dynamics were evaluated using a linear mixed-effects model with restricted maximum likelihood. Time and exposure status, as categorical values, were included as fixed effects. The individual donor ID was included as a random effect nested with "exposure status." Age and sex were initially included in the model but were not found to improve the model AIC and were therefore excluded. Following model evaluation, specific comparisons between previously exposed or primary infected individuals were done with a least squares mean differences Student's $t$ test. Comparisons were presented as fold difference with a $95 \%$ CI.

Study approval. Written informed consent was obtained from all participants. The study was approved by the Regional Ethical Committee in Stockholm.

\section{Author contributions}

CS, CR, AA, MNF, KEMP, PB, and AF researched the concept. CS, CR, VY, PJ, TL, KS, MA, and AF performed the experiments. CS, CR, YC, and JM analyzed data. CS performed statistical analysis 
in consultation with VY and MA. CS, CR, and AF wrote the first draft of the paper, and all authors contributed in the writing and revision of the manuscript.

\section{Acknowledgments}

Our most sincere gratitude to all study individuals contributing repeated blood samples. We thank Christine Stenström and colleagues at the Department of Clinical Microbiology as well as research nurses and clinicians at Karolinska University Hospital for assistance during sample collection. We also thank Fariba Foroogh and team members for technical assistance and Ulf Hammar for statistical consultation. We thank John Mascola and Nicole Doria-Rose for providing 3T3-msCD40L feeder cells. This work was supported by funds from Stockholm County Council $(20130207,20150135)$ and the Swedish Research Council (248-2013-6573, 2015-02977), the Marianne and Marcus Wallenberg Foundation (2010-0067) to AF, the Stockholm County Council (K1414-2012-4) to CR, Karolinska Institutet Funds and Foundations (2016fobi50116), the Jonas Söderquist award, and the Magnus Bergvall Foundation (2017-02043) to CS.

Address correspondence to: Christopher Sundling, Bioclinicum J7:20, Visionsgatan 4, 17164 Solna, Sweden. Phone: 46.8.524.800.00; Email: christopher.sundling@ki.se.

1. WHO. World Malaria Report 2018. https://www.who.int/malaria/publications/world-malaria-report-2018/report/en/. Accessed April 25, 2019

2. Langhorne J, Ndungu FM, Sponaas AM, Marsh K. Immunity to malaria: more questions than answers. Nat Immunol. 2008;9(7):725-732.

3. Cohen S, Mcgregor IA, Carrington S. Gamma-globulin and acquired immunity to human malaria. Nature. 1961;192:733-737.

4. Triller G, et al. Natural Parasite Exposure Induces Protective Human Anti-Malarial Antibodies. Immunity. 2017;47(6):1197-1209.e10.

5. Weiss GE, et al. The Plasmodium falciparum-specific human memory B cell compartment expands gradually with repeated malaria infections. PLoS Pathog. 2010;6(5):e1000912.

6. Mugyenyi CK, et al. Declining Malaria Transmission Differentially Impacts the Maintenance of Humoral Immunity to Plasmodium falciparum in Children. J Infect Dis. 2017;216(7):887-898.

7. Ndungu FM, Lundblom K, Rono J, Illingworth J, Eriksson S, Färnert A. Long-lived Plasmodium falciparum specific memory B cells in naturally exposed Swedish travelers. Eur J Immunol. 2013;43(11):2919-2929.

8. Nogaro SI, et al. The breadth, but not the magnitude, of circulating memory B cell responses to P. falciparum increases with age/exposure in an area of low transmission. PLoS One. 2011;6(10):e25582.

9. Tan J, et al. A LAIR1 insertion generates broadly reactive antibodies against malaria variant antigens. Nature. 2016;529(7584):105-109.

10. Illingworth J, et al. Chronic exposure to Plasmodium falciparum is associated with phenotypic evidence of B and T cell exhaustion. J Immunol. 2013;190(3):1038-1047.

11. Portugal S, et al. Malaria-associated atypical memory B cells exhibit markedly reduced B cell receptor signaling and effector function. Elife. 2015;4:e07218.

12. Obeng-Adjei N, et al. Circulating Th1-Cell-type Tfh Cells that Exhibit Impaired B Cell Help Are Preferentially Activated during Acute Malaria in Children. Cell Rep. 2015;13(2):425-439.

13. Ryg-Cornejo V, et al. Severe Malaria Infections Impair Germinal Center Responses by Inhibiting T Follicular Helper Cell Differentiation. Cell Rep. 2016;14(1):68-81.

14. Scholzen A, et al. BAFF and BAFF receptor levels correlate with B cell subset activation and redistribution in controlled human malaria infection. J Immunol. 2014;192(8):3719-3729.

15. Obeng-Adjei N, et al. Malaria-induced interferon- $\gamma$ drives the expansion of Tbethi atypical memory B cells. PLoS Pathog 2017;13(9):e1006576.

16. Weiss GE, et al. Atypical memory B cells are greatly expanded in individuals living in a malaria-endemic area. $J$ Immunol. 2009;183(3):2176-2182.

17. Ubillos I, et al. Chronic Exposure to Malaria Is Associated with Inhibitory and Activation Markers on Atypical Memory B Cells and Marginal Zone-Like B Cells. Front Immunol. 2017;8:966.

18. Sullivan RT, et al. FCRL5 Delineates Functionally Impaired Memory B Cells Associated with Plasmodium falciparum Exposure. PLoS Pathog. 2015;11(5):e1004894.

19. Muellenbeck MF, et al. Atypical and classical memory B cells produce Plasmodium falciparum neutralizing antibodies. $J$ Exp Med. 2013;210(2):389-399.

20. Rubtsov AV, Rubtsova K, Kappler JW, Jacobelli J, Friedman RS, Marrack P. CD11c-Expressing B Cells Are Located at the T Cell/B Cell Border in Spleen and Are Potent APCs. J Immunol. 2015;195(1):71-79.

21. Rivera-Correa J, et al. Plasmodium DNA-mediated TLR9 activation of T-bet. Nat Commun. 2017;8(1):1282.

22. Weiss GE, et al. A positive correlation between atypical memory B cells and Plasmodium falciparum transmission intensity in cross-sectional studies in Peru and Mali. PLoS One. 2011;6(1):e15983.

23. Portugal S, et al. B cell analysis of ethnic groups in Mali with differential susceptibility to malaria. Malar J. 2012;11:162.

24. Lugaajju A, Reddy SB, Wahlgren M, Kironde F, Persson KE. Development of Plasmodium falciparum specific naïve, atypical, memory and plasma B cells during infancy and in adults in an endemic area. Malar J. 2017;16(1):37. 
25. Joosten SA, et al. Patients with Tuberculosis Have a Dysfunctional Circulating B-Cell Compartment, Which Normalizes following Successful Treatment. PLoS Pathog. 2016;12(6):e1005687.

26. Moir S, et al. Evidence for HIV-associated B cell exhaustion in a dysfunctional memory B cell compartment in HIV-infected viremic individuals. J Exp Med. 2008;205(8):1797-1805.

27. Wei $\mathrm{C}$, et al. A new population of cells lacking expression of CD27 represents a notable component of the B cell memory compartment in systemic lupus erythematosus. J Immunol. 2007;178(10):6624-6633.

28. Isnardi I, et al. Complement receptor 2/CD21- human naive B cells contain mostly autoreactive unresponsive clones. Blood. 2010;115(24):5026-5036.

29. Terrier B, et al. Expansion of functionally anergic CD21-/low marginal zone-like B cell clones in hepatitis C virus infection-related autoimmunity. J Immunol. 2011;187(12):6550-6563.

30. Saadoun D, et al. Expansion of autoreactive unresponsive CD21-/low B cells in Sjögren's syndrome-associated lymphoproliferation. Arthritis Rheum. 2013;65(4):1085-1096.

31. Rakhmanov M, et al. Circulating CD21low B cells in common variable immunodeficiency resemble tissue homing, innate-like B cells. Proc Natl Acad Sci USA. 2009;106(32):13451-13456.

32. Jacobi AM, et al. Activated memory B cell subsets correlate with disease activity in systemic lupus erythematosus: delineation by expression of CD27, IgD, and CD95. Arthritis Rheum. 2008;58(6):1762-1773.

33. Yman V, et al. Antibody responses to merozoite antigens after natural Plasmodium falciparum infection: kinetics and longevity in absence of re-exposure. BMC Med. 2019;17(1):22.

34. Levine JH, et al. Data-Driven Phenotypic Dissection of AML Reveals Progenitor-like Cells that Correlate with Prognosis. Cell. 2015;162(1):184-197.

35. Sanz I, Wei C, Lee FE, Anolik J. Phenotypic and functional heterogeneity of human memory B cells. Semin Immunol. 2008;20(1):67-82

36. Wu YC, Kipling D, Dunn-Walters DK. The relationship between CD27 negative and positive B cell populations in human peripheral blood. Front Immunol. 2011;2:81

37. Sims GP, Ettinger R, Shirota Y, Yarboro CH, Illei GG, Lipsky PE. Identification and characterization of circulating human transitional B cells. Blood. 2005;105(11):4390-4398.

38. Myles A, Gearhart PJ, Cancro MP. Signals that drive T-bet expression in B cells. Cell Immunol. 2017;321:3-7.

39. Daniel Ribeiro C. Polyclonal B-cell activation (PBA). What have we learned from the study of malaria? Mem Inst Oswaldo Cruz. 1988;83 Suppl 1:633-648.

40. Aurrecoechea C, et al. PlasmoDB: a functional genomic database for malaria parasites. Nucleic Acids Res. 2009;37(Database issue):D539-D543.

41. Aranburu A, et al. Age-associated B cells expanded in autoimmune mice are memory cells sharing H-CDR3-selected repertoires. Eur J Immunol. 2018;48(3):509-521.

42. Zinöcker $\mathrm{S}$, et al. The $\mathrm{V}$ gene repertoires of classical and atypical memory B cells in malaria-susceptible West African children. $J$ Immunol. 2015;194(3):929-939.

43. Bagnara D, et al. A Reassessment of IgM Memory Subsets in Humans. J Immunol. 2015;195(8):3716-3724.

44. King T, Lamb T. Interferon- $\gamma$ : The Jekyll and Hyde of Malaria. PLoS Pathog. 2015;11(10):e1005118.

45. Lau D, et al. Low CD21 expression defines a population of recent germinal center graduates primed for plasma cell differentiation. Sci Immunol. 2017;2(7):null.

46. Inoue T, Moran I, Shinnakasu R, Phan TG, Kurosaki T. Generation of memory B cells and their reactivation. Immunol Rev. 2018;283(1):138-149.

47. Ehrhardt GR, et al. Expression of the immunoregulatory molecule FcRH4 defines a distinctive tissue-based population of memory B cells. J Exp Med. 2005;202(6):783-791.

48. Ehrhardt GR, Hijikata A, Kitamura H, Ohara O, Wang JY, Cooper MD. Discriminating gene expression profiles of memory B cell subpopulations. J Exp Med. 2008;205(8):1807-1817.

49. Asghar M, et al. Cellular aging dynamics after acute malaria infection: A 12-month longitudinal study. Aging Cell. 2018;17(1):e12702.

50. Vafa Homann M, et al. Detection of Malaria Parasites After Treatment in Travelers: A 12-months Longitudinal Study and Statistical Modelling Analysis. EBioMedicine. 2017;25:66-72.

51. Shokoples SE, Ndao M, Kowalewska-Grochowska K, Yanow SK. Multiplexed real-time PCR assay for discrimination of Plasmodium species with improved sensitivity for mixed infections. J Clin Microbiol. 2009;47(4):975-980.

52. Chen H, Lau MC, Wong MT, Newell EW, Poidinger M, Chen J. Cytofkit: A Bioconductor Package for an Integrated Mass Cytometry Data Analysis Pipeline. PLoS Comput Biol. 2016;12(9):e1005112.

53. Huang J, et al. Isolation of human monoclonal antibodies from peripheral blood B cells. Nat Protoc. 2013;8(10):1907-1915

54. Osier FH, et al. Breadth and magnitude of antibody responses to multiple Plasmodium falciparum merozoite antigens are associated with protection from clinical malaria. Infect Immun. 2008;76(5):2240-2248. 